'Departamento de Salud Pública. ${ }^{2}$ Departamento de Psiquiatría.

${ }^{3}$ Departamento de Neurología y Centro de Bioética.

${ }^{4}$ Departamento de Endocrinología y Centro de Bioética, Pontificia Universidad Católica de Chile.

Proyecto financiado por fondos de la Vicerrectoría Adjunta de Investigación y Doctorado. Dirección General de Pastoral y Cultura Cristiana, de la Pontificia Universidad Católica de Chile a través del concurso "120 años nos comprometen". La Vicerrectoría no tuvo influencia en ninguna de las etapas del estudio.

Recibido el 15 de mayo de 2012, aceptado el 21 de diciembre de 2012.

Correspondencia a: Dra. Paula Bedregal. MPH, PhD Departamento de Salud Pública. Escuela de Medicina. Pontificia Universidad Católica de Chile. Marcoleta 434, Santiago, Chile. Teléfono: 056-2-354 6893 Celular: 9-2391530

E-mail: pbedrega@med.puc.cl

\section{Criterios de juicio moral de estudiantes de Medicina, según el Modelo Intuitivo Social}

\author{
PAULA BEDREGAL ${ }^{1}$, TOMÁS LEÓN², \\ BEATRIZ SHAND ${ }^{3}$, LORENA MOSSO ${ }^{4}$
}

\section{Survey on moral judgment criteria among medical students}

Background: The evaluation of moral judgment criteria in medical students is important to develop effective educational programs in bioethics. Aim: To compare priority judgment criteria and moral judgment tendency in medical students of first and fifth grade. Material and Methods: The Moral Foundations Questionnaire (MFQ30), to identify moral criteria was applied to 259 students, 63.7\% from first year and 50.2\% women. Results: The dominant moral tendency both in first and fifth year students was liberal. Justice and compassion were the most important criteria in men and in women, respectively. Respect towards authority and sanctity were the least important criteria in women and men, respectively. Conclusions: The implications for moral psychology and medical education of these results are discussed.

(Rev Med Chile 2013; 141: 704-709).

Key words: Bioethics; Decision making; Moral development.
$\mathrm{D}$ esde que la bioética se instala como disciplina en la formación médica, ha habido interés en conocer cuáles son los criterios de juicio moral utilizados por médicos y estudiantes y cómo es posible educar el juicio moral ${ }^{1,2}$.

En la educación moral, se identifican al menos dos perspectivas: la educación del carácter moral y la educación del razonamiento moral ${ }^{3,4}$. La primera se basa en la noción de virtudes de Aristóteles, las que disponen al sujeto a un hábito de acción ${ }^{5}$. Lickona actualiza esta perspectiva, y define al carácter moral compuesto por tres aspectos: el conocimiento moral, la voluntad moral y la acción moral ${ }^{6}$. La formación del carácter moral se lograría mediante la adquisición de virtudes a través de la práctica de hábitos o maneras de proceder fundamentadas en valores como: el respecto, la responsabilidad la honestidad, la justicia, el cuidado, y el civismo ${ }^{6}$. La segunda tiene como figura central a Kohlberg, quien se basa en Kant respecto de la búsqueda de una teoría universal, racional y normativa de la moral, y en Piaget, respecto de un enfoque constructivista del desarrollo moral ${ }^{7}$. Este último aspecto es el que origina la formulación de las seis etapas del desarrollo del razonamiento moral ${ }^{8}$. Para este autor, los criterios morales fundamentales -en los que descansa este razonamiento moral- son la justicia, igualdad y equidad; la formación de la moralidad se basaría en comprender mejor estos criterios, lo que permite el desarrollo moral. Kohlberg ha sido criticado por: priorizar la justicia por sobre otros valores, basarse en observaciones en varones ${ }^{9}$, y por considerar sólo los aspectos cognitivos conscientes ${ }^{10}$.

Haidt, propone ir hacia una educación moral, basada en el modelo "intuitivo social o de sentido moral", el que se fundamenta en evidencias científicas sobre el papel de las emociones y automatismos en la valoración de sucesos ${ }^{10-12}$. En este modelo, las emociones serían un primer marcador que, conjunto con el razonamiento moral, permitirían el juicio moral. Las intuiciones serían ideas 
generadas por procesos automáticos, rápidos, los que se integrarían con el conocimiento aprendido acerca del mundo social, para determinar un juicio. Diferenciar entre el bien y mal sería innato en el ser humano, y el aprendizaje (educación moral) desde las primeras etapas del desarrollo, permitiría adquirir un repertorio de contenidos y conductas frente a situaciones específicas. Para determinar el tipo de intuiciones que guía el juicio moral, Haidt se basó en los tres sustratos éticos de Shweder ${ }^{13}$ (autonomía, comunidad y divinidad) que describen los códigos morales observados en diversas culturas, y en la teoría de los modelos relacionales de Fiske ${ }^{14}$.

En el modelo de Haidt, los criterios de juicio moral corresponden a cinco grupos de intuiciones morales innatas, cuya expresión en el actuar moral depende del ejercicio de las virtudes con las que se asocian ${ }^{11}$. Desde esta perspectiva, las virtudes serían habilidades sociales adquiridas mediante el entrenamiento de las propias facultades innatas. Haidt se basó en estudios realizados en primates y humanos, los que han mostrado que en ambas especies se manifiestan tres criterios de juicio moral ${ }^{15}$ :

1. Frente al sufrimiento el criterio de compasión, el que permitiría la supervivencia de la especie.

2. Frente a la justicia el criterio de reciprocidad, que permitiría el desempeño social, la convivencia, la aceptación de otro y la reproducción.

3. Frente a la autoridad o jerarquía el criterio de respeto, que permitiría el ordenamiento de la convivencia social, la organización y desarrollo del grupo.

Dos criterios más, propios del ser humano y que se observan en todas las culturas son ${ }^{15}$ :

1. Frente a la preservación del propio grupo, el criterio de lealtad, que implica la identificación personal con un grupo y el resguardo de códigos sociales que permiten la interacción específica en éste.

2. La preocupación por el desarrollo y pureza del propio cuerpo, que implica cuidar la alimentación y la higiene corporal para sobrevivir, y también su sacralidad. Esto se expresa en ciertas creencias respecto al sexo, a la comida, al embarazo, la menstruación y el manejo de los cadáveres, lo que se observa en prácticamente todas las culturas, y sólo incipientemente en algunos mamíferos que han tenido proximidad con los seres humanos ${ }^{16}$.
Con base a estos criterios, Haidt y cols proponen la construcción de un perfil de tendencias de comportamiento moral: liberal y conservador ${ }^{17}$. El primero fundamenta su actuar en los criterios de justicia y compasión; mientras que el segundo en el equilibrio de los cinco criterios.

El objetivo de este estudio es conocer y comparar los criterios prioritarios de juicio moral de estudiantes de medicina de primero y de quinto año (que ya cursaron las asignaturas obligatorias de bioética) y la tendencia de juicio moral, según al modelo de Haidt.

\section{Material y Método}

Se realizó un estudio observacional transversal en estudiantes de primero y quinto año de Medicina de la Pontificia Universidad Católica de Chile. El estudio fue aprobado por el Comité de Ética de Investigación de la Escuela de Medicina.

La muestra mínima estimada con potencia de $80 \%$, error I de $5 \%$ y diferencias entre grupos de $50 \%$, fue de al menos 90 por cada grupo (primero y quinto), de modo que la muestra total fuera de 180. El universo disponible era de 347 alumnos, $61 \%$ hombres. La muestra alcanzada fue de 259 alumnos, 63,7\% de primer año. Cincuenta y siete como seis por ciento eran mujeres en primer año, mientras que en quinto sólo $37,2 \%$. El promedio de edad para los alumnos de primer año fue de 18,9 años $(\mathrm{DE}=1,27)$ y en quinto fue de 22,6 años $(\mathrm{DE}=1,32)$.

La variable dependiente es el criterio de juicio moral y la tendencia moral (más conservador o más liberal) según el modelo de Haidt. Las variables independientes exploradas fueron el sexo y el curso.

El instrumento utilizado es el Moral Foundations Questionnaire: 30-Item Full Versión (MFQ30). Este es un cuestionario autoaplicado, de 30 ítems distribuidos en dos áreas, la primera interroga sobre la relevancia de diversos criterios para juzgar una acción como buena o mala, y la segunda indaga sobre el grado de acuerdo con aseveraciones sobre situaciones específicas, todos medidos con escala tipo Likert de 6 puntos (0-5). A mayor puntaje, mayor acuerdo con el criterio o aseveración. El instrumento fue diseñado y validado en sus constructos ${ }^{20,22}$. La versión hispana utilizada en este estudio contó con la autorización 
del autor y fue traducido ida y vuelta de manera de asegurar la comprensión y semejanza con la versión inglesa. La versión final para libre uso, se encuentra en la página web www.moralfoundations.org. Su aplicación se realizó en horario de clases y fue de carácter voluntario, previa firma de consentimiento informado por parte de los estudiantes. Su duración promedio fue de $15 \mathrm{~min}$. Su consistencia interna se muestra en la Tabla 1.

Se realizó un análisis estadístico descriptivo de los criterios más frecuentes encontrados, y la tendencia de juicio, para todo el grupo y para cada subgrupo. El promedio de importancia de cada criterio se comparó entre grupos utilizando métodos paramétricos y no paramétricos según corresponda, al igual que las frecuencias. Se consideró como diferencias significativas con un error tipo I de $<0,05$. El análisis y procesamiento se realizó con el software de análisis estadístico SPSS 16.0.

\section{Resultados}

El criterio moral más importante encontrado fue compasión, seguido por reciprocidad (justi- cia), siendo ambos igualmente relevantes para los alumnos de primer año y quinto año. En los dos grupos, el menos importante fue el respeto por la autoridad. Se encuentran diferencias estadísticamente significativas en los promedios en los criterios de lealtad hacia el propio grupo y pureza corporal entre los alumnos de primero y quinto año, siendo ambos criterios más importantes en los menores (Tabla 2).

En el análisis por sexo (Tabla 3), el criterio más relevante en mujeres fue la compasión, mientras que en hombres la reciprocidad (justicia); en mujeres el menos relevante fue el respeto a la autoridad, mientras en hombres fue la pureza corporal.

La tendencia moral preponderante en los estudiantes de medicina encuestados, fue liberal $(86,1 \%)$; este porcentaje fue similar en alumnos de primero y de cursos superiores. En el caso de las mujeres el porcentaje con perfil liberal fue de $88,5 \%$ y en hombres de $83,7 \%$, sin diferencias estadísticamente significativas.

Cincuenta y cinco alumnos $(21,2 \%)$ no estuvieron de acuerdo con el ítem "Nunca es correcto matar a un ser humano" (puntaje $\leq 3$ ). No se encontró diferencias estadísticamente significa-

Tabla 1. Consistencia interna, puntaje promedio y rango observado MFQ30

\begin{tabular}{|lccc|}
\hline Criterio de juicio & Alfa Cronbach & Promedio observado & Rango \\
\hline Compasión & 0,582 & 3,92 & $3,61-4,21$ \\
Reciprocidad & 0,631 & 3,89 & $2,08-4,56$ \\
Lealtad al grupo & 0,587 & 3,38 & $1,82-4,16$ \\
\hline Sacralidad corporal & 0,752 & 2,96 & $2,50-3,40$ \\
Respeto a la autoridad & 0,610 & 2,83 & $1,97-4,01$ \\
\hline
\end{tabular}

Tabla 2. Puntaje promedio en criterios de juicio moral y su relevancia, por curso

\begin{tabular}{|c|c|c|c|c|c|c|}
\hline \multirow[t]{2}{*}{ Criterio } & \multicolumn{3}{|c|}{ Promedio puntaje (0-5) } & \multicolumn{3}{|c|}{$\begin{array}{l}\% \text { del total de alumnos que calificó } \\
\text { como muy relevante el criterio }\end{array}$} \\
\hline & Total & Primer año & Quinto Año & Total & Primer año & Quinto año \\
\hline Compasión & 3,91 & 3,93 & 3,90 & 81,1 & 83,0 & 86,2 \\
\hline Reciprocidad & 3,89 & 3,93 & 3,81 & 83,4 & 84,8 & 80,9 \\
\hline Lealtad al grupo*,\& & 3,33 & 3,40 & 3,21 & 44,4 & 49,1 & 36,2 \\
\hline Sacralidad corporal*,\& & 2,94 & 3,05 & 2,75 & 35,1 & 38,8 & 28,7 \\
\hline Respeto a la autoridad & 2,83 & 2,81 & 2,87 & 23,9 & 24,2 & 23,4 \\
\hline
\end{tabular}

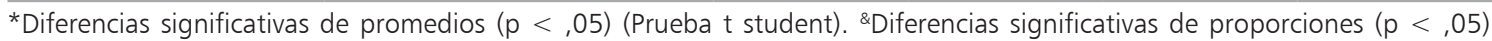
(Prueba z). 
Criterios de juicio moral en estudiantes de Medicina - P. Bedregal et al

Tabla 3. Puntaje promedio en criterios de juicio moral y su relevancia, por sexo

\begin{tabular}{|c|c|c|c|c|c|c|}
\hline \multirow[t]{2}{*}{ Criterio } & \multicolumn{3}{|c|}{ Promedio puntaje (0-5) } & \multicolumn{3}{|c|}{$\begin{array}{l}\text { \% del total de alumnos que calificó } \\
\text { como muy relevante el criterio }\end{array}$} \\
\hline & Total & Mujeres & Hombres & Total & Mujeres & Hombres \\
\hline Compasión*\& & 3,91 & 4,08 & 3,76 & 81,1 & 90,0 & 72,1 \\
\hline Reciprocidad & 3,89 & 3,94 & 3,83 & 83,4 & 85,4 & 81,4 \\
\hline Lealtad al grupo* & 3,33 & 3,41 & 3,25 & 44,4 & 49,2 & 39,5 \\
\hline Sacralidad corporal* & 2,94 & 3,12 & 2,77 & 35,1 & 37,7 & 32,6 \\
\hline Respeto a la autoridad & 2,83 & 2,87 & 2,79 & 23,9 & 24,6 & 23,3 \\
\hline
\end{tabular}

*Diferencias significativas entre promedios con $\mathrm{p}<, 05$ (prueba t-student). ${ }^{\&}$ Diferencias significativas entre proporciones con $p<, 05$ (Prueba Z).

tivas en la proporción por curso, pero sí por sexo $(\mathrm{p}<, 05)$. Entre los que no estuvieron de acuerdo, $67 \%$ eran hombres. Hay una correlación directa entre el grado de acuerdo con el ítem y el criterio de compasión $(\mathrm{r}=0,41 ; \mathrm{p}<, 05)$.

Ciento quince alumnos $(44,4 \%)$ no estuvieron de acuerdo con el ítem "Es más importante ser jugador de un equipo que jugar solo". No hubo diferencias estadísticamente significativas por sexo. Sin embargo, hubo una proporción mayor de alumnos de quinto año que no estuvieron de acuerdo con ese ítem (47\%) que los de primer año $(36,8 \%)(\mathrm{p}<, 05)$. Aquellos alumnos que no estuvieron de acuerdo con el ítem, en 36,8\% estimaron muy relevante el criterio de lealtad hacia el propio grupo, mientras que $79,1 \%$ de aquellos que estaban de acuerdo con el ítem lo estimaron relevante $(\mathrm{p}<, 05)$. Por otra parte, aquellos que no estuvieron de acuerdo con el ítem, en 57,6\% estimaron muy relevante el criterio de sacralidad corporal, mientras que $73,9 \%$ de aquellos que estuvieron de acuerdo con el ítem estimaron relevante ese criterio $(\mathrm{p}<, 05)$.

Finalmente, la baja importancia otorgada por todos los estudiantes a la pureza corporal se asoció con una baja valoración al criterio de autoridad $(\mathrm{r}=0,44, \mathrm{p}<, 05)$.

\section{Discusión}

Este estudio se plantea desde el modelo intuitivo social de Haidt. Reconociendo las limitaciones de una aproximación puramente psicológica de la moralidad, proponemos este modelo como un aporte para la reflexión sobre los criterios mo- rales de estudiantes universitarios. Este modelo permite plantear que la moralidad humana no sólo se expresa en términos de justicia y cuidado sino que existen otros aspectos relevantes que se asocian a otros modos de comprender el mundo en diferentes grupos y culturas, en que la lealtad al propio grupo, el respeto a la autoridad y pureza corporal son relevantes.

La aplicación de MFQ30 en este estudio mostró lo esperado y observado en otros grupos occidentales: el predominio de una tendencia liberal, en que priman el criterio de justicia y la compasión ${ }^{18}$. El análisis por sexo reveló una mayor valoración del criterio de compasión por parte de las mujeres que de los hombres, coherente con los postulados de Gilligan ${ }^{9}$, similar a un meta-análisis, que muestra una leve orientación hacia el cuidado por parte de las mujeres $(\mathrm{d}=-, 28)$ y de orientación hacia la justicia en hombres $(d=0,19)$ que no alcanza significación estadística ${ }^{19}$. Es probable que, independiente de posibles diferencias innatas al respecto, la mayor valoración por la compasión/justicia pueda ser atribuible a un reforzamiento cultural. Este aspecto puede estar cambiando en algunas sociedades, al equilibrarse los roles masculinos y femeninos. Para indagar al respecto sería necesario estudios que comparen diversas cohortes de edad de estudiantes y médicos, ajustados por los roles de género efectivamente ejercidos por las personas.

Llamó la atención la baja relevancia otorgada al criterio de respeto a la autoridad y sacralidad corporal. Ambos conceptos tienen sus bases en el ámbito religioso e histórico-cultural ${ }^{20}$. En el primer caso, hay evidencias de que adolescentes chilenos otorgan menos legitimidad a la autoridad de sus padres y se sienten menos obligados a obedecer 
normas con las que no concuerdan, comparados con adolescentes filipinos o estadounidenses ${ }^{21}$. La sacralidad corporal ha sido poco estudiada en los adolescentes; sin embargo, hay evidencias de diferencias culturales al respecto. La menor sacralidad corporal se asocia con conductas morales fuera de la norma en espacios virtuales (internet) y con baja adscripción religiosa ${ }^{22}$.

El análisis por grupo de edad mostró que los alumnos de primero, tuvieron más respuestas favorables hacia el criterio de lealtad hacia el grupo que los de quinto. Este aspecto contradice la propuesta educativa en medicina de adquirir habilidades para el trabajo grupal. Es posible que el conocimiento sobre cómo trabajar en grupo se adquiera, pero la actitud pro-grupo puede que no esté asentada o se asocie a la importancia que los jóvenes le otorgan al grupo de iguales, el que se reduce con el paso de la adolescencia temprana hacia la adultez. También es posible que su importancia se reduzca si la valoración del grupo se desincentiva en la práctica ${ }^{23}$.

Otros estudios utilizando instrumentos construidos con otra teoría (Lind), han encontrado una reducción en las competencias morales a través de los años de estudio de medicina ${ }^{24-26}$. Esto apoyaría el que ciertos criterios de juicio imperen más sobre otros al pasar los años de entrenamiento médico, los que pueden relacionarse con cambios propios del paso de la adolescencia a la juventud, además de los incentivos del ambiente de aprendizaje ${ }^{24-26}$.

El modelo propuesto por Haidt pretende ser descriptivo y explicativo respecto de las conductas humanas ${ }^{27}$. Críticos del modelo plantean que si bien los criterios son apropiados y sustentables empíricamente, pareciera ser que la relación entre un perfil o criterio predominante en el sujeto no necesariamente se asocia con la acción moral ${ }^{27}$. En este sentido, existe evidencia científica que sugiere que la identidad moral del sujeto explica mayormente la acción moral y es la que motiva la acción $^{28}$. De modo que se propone que las teorías de desarrollo moral que incluyan la identidad moral serían superiores a aquellas basadas en la cognición o los afectos ${ }^{27}$.

La investigación hasta la fecha es contundente en señalar que un modelo educativo basado exclusivamente en el razonamiento moral individual no permitiría lograr modificaciones en las conductas morales o el desarrollo moral pleno. Desde un modelo de educación del carácter, como el pro- puesto por Lickona, basado en trabajo individual y grupal, que considera el cultivo de las virtudes en tres niveles (conocimiento, sentimiento, y conducta), es posible verificar cambios ${ }^{6}$. Narváez ha propuesto un modelo integrado de educación ética que utiliza los presupuestos de Lickona, y las evidencias científicas desde la neurociencia y la psicología social, y que incorpora el concepto de novicios y expertos morales, estimando que la práctica permite ganar el "know-how" ético; sin embargo, en su conceptualización se reduce el papel de las intuiciones morales, del sí mismo moral y del colectivo ${ }^{26}$.

Finalmente, la educación moral, considerando a Haidt, implicaría reforzar las intuiciones morales de nuestros estudiantes, que son deseables para el ejercicio médico, y favorecer el entrenamiento de su razonamiento moral; sin descuidar reforzar aquellos aspectos de identidad que le hacen constituirse en un ser moral ${ }^{27}$. Para esto no basta con una educación individualizada sino que se requiere de una comunidad de aprendizaje en la cual la identidad y los valores a reforzar se expresen y se motiven ${ }^{3,29}$.

\section{Referencias}

1. Culver R, Clouser R, Gert B, Brody H, Fletcher J, Jonsen A, et al. Basic curricular goals in medical ethics. N Engl J Med 1985; 312: 253-6.

2. Swick, H, Szenas P, Danoff, D. Whitcomb ME. Teaching Professionalism in undergraduate medical education. JAMA. 1999; 282: 830-2.

3. Graham J, Haidt J, Rimm-Kaufman S. Ideology and intuition in moral education. Eur J Dev Sci 2008; 2: 26986.

4. Vargas L, González-Torres MC. La revitalización de la educación del carácter en el ámbito psicoeducativo actual: aportaciones desde las ciencias de la prevención y la psicología positiva. Electronic Journal of Research in Educational Psychology 2009; 7: 1379-418.

5. Lapsley DK, Narváez D. A Social-cognitive approach to the moral personality. En D. K. Lapsley y D. Narváez, Editores, Moral development, self and identity. Mahwah, NJ: Erlbaum, 2004; p. 189-212.

6. Lickona T. The return of character education. Educational Leadership 1993; 51: 6-11.

7. Kohlberg L. The psychology of moral development. New York, Estados Unidos: Editorial Harper \& Row. 1984.

8. Kohlberg L. Stage and sequence: the cognitive-developmental approach to socialization. En DA. Goslin, Editor, 
Handbook of socialization theory and research. Chicago: Rand McNally; 1969. p. 347-480.

9. Gilligan C. In a different voice. Cambridge, Estados Unidos: Editorial Harvard University Press. 1982.

10. Greene J, Haidt J. How (and where) does moral judgment work? Trends Cogn Sci 2002; 6: 517-23.

11. Haidt J, Joseph C. Intuitive ethics: How innately prepared intuitions generate culturally variable virtues. Daedalus, 2004; 3: 55-66.

12. Haidt J. The emotional dog and its rational tail: A social intuitionist approach to moral judgment. Psychol Rev 2001; 108: 814-34.

13. Shweder RA. Beyond self-constructed knowledge: The study of culture and morality. Merrill-Palmer Q 1982; 28: 41-69.

14. Fiske A. Four elementary forms of sociality: Framework for a unified theory of social relations. Psychol Rev 1992; 99: 689-723.

15. De Waal F. Good natured: The origins of right and wrong in humans and other animals. Cambrigde MA. Estados Unidos: Editorial Harvard University Press. 1996.

16. Haidt J, Hersh Ma. Sexual morality: The cultures and reason of liberals and conservatives. J Appl Soc Psychol 2001; 31: 191-221.

17. Haidt J, Graham J. When morality opposes justice: Conservatives have moral intuitions that liberals may not recognize. Social Justice Research 2007; 20: 98-116.

18. Graham J, Haidt J, Nosek B. Liberals and conservatives use different sets of moral foundations. J Pers Soc Psychol 2009; 96: 1029-46.

19. Jaffee S, Hyde JS. Gender differences in moral orientation: A meta-analysis. Psychol Bull 2000; 126: 703-26.

20. Jensen LA. Through two lenses: A cultural-developmental approach to moral psychology. Dev Rev 2008; 28: 289-315.
21. Darling N, Cumsille P, Peña-Alampay L. Rules, legitimacy of parental authority, and obligation to obey in Chile, the Philippines, and the United States. En Smetana J, Editora, Changing boundaries of parental authority during adolescence. San Francisco, Estados Unidos: Editorial Jossey-Bass; 2005. p. 47-60.

22. Joeckel S, Bowman ND, Dogruel L. Gut or game: The influence of moral intuitions on decisions in virtual environments. Media Psychology 2012; 15: 372-8.

23. Lerner $S$, Magrane D, Friedman E. Teaching team work in medical education. Mt Sinai J Med 2009; 76: 318-29.

24. Wolf TM, Balson PM, Faucet JM. A retrospective study of attitudes change during medical education. Med Educ 1989; 23: 19-23.

25. Lind G. Are helpers always moral? Empirical findings from a longitudinal study of medical students in Germany. Proceedings of the Regional Conference of the International Council of Psychologists: Moral development and prosocial behavior; 1997 July; Padua, Italy. ebookbrowse.com/lind-2000-are-helpers-always-moral

26. Narváez D. How cognitive and neurobiological sciences inform values education for creatures like us. En Aspin D, Chapman JD, Editores, Values education and lifelong learning: philosophy, policies, programmes. Dordrecht, Holanda: Editorial Springer Press International; 2008. p. 127-.

27. Musshenga A. Moral Judgment and moral reasoning. A critique of Jonathan Haidt. En: Düwell M, RehmannSutter C, Mieth D, Editores, The contingent nature of life. Dordrecht: Springer Verlag; 2008. p. 131-40.

28. Hardy SA, Carlo G. Identity as a source of moral motivation. Hum Dev 2005; 48: 232-56.

29. Solovackova B, Slovacek L. Moral Judgment Competence and Moral Attitudes of Medical Students. Nurs Ethics 2007; 14: 320-8. 